\title{
Laparoscopic middle pancreatectomy under a pancreatic duct-navigation surgery
}

Amane Kitasato, M.D., Tomohiko Adachi, M.D., Takamitsu Inokuma, M.D., Yoshitsugu Tajima, M.D., Takashi Kanematsu, M.D., Tamotsu Kuroki, M.D.

Department of Surgery, Nagasaki University, Graduate School of Biomedical Sciences, 1-7-1 Sakamoto, Nagasaki 852-8501, Japan

Correspondence to: Amane Kitasato, Department of Surgery, Nagasaki University, Graduate School of Biomedical Sciences, 1-7-1 Sakamoto, Nagasaki 852-8501, Japan Telephone: +81-95-8197316 Fax: +81-95-8197319

E-mail: a-kit@bg8.so-net.ne.jp

Running title: Laparoscopic middle pancreatectomy

Key words: Laparoscopic surgery, Pancreatectomy, Middle pancreatectomy, Minimally invasive surgery, Pancreatic duct-navigation surgery

Abbreviations: IPMNs: intraductal papillary mucinous neoplasms, MPD: main pancreatic duct, CT: computed tomography, MRP: magnetic resonance pancreatography, ERP: endoscopic retrograde pancreatography, ENPD: endoscopic naso-pancreatic drainage 
Kitasato

\section{Summary}

Recent developments of diagnostic modalities have increased detection of many benign and low malignant pancreatic lesions, and then various minimally invasive surgeries and/or pancreatic function preserving surgeries have been devised for such lesions. Laparoscopic pancreatic surgery has developed rapidly in recent years, and it’s minimally invasiveness is expected. This report describes a laparoscopic middle pancreatectomy under a pancreatic duct-navigation surgery for a localized main pancreatic duct stenosis, and this method is effective to benign and low malignant pancreatic lesions in point of minimally invasiveness and function preservation. 


\section{Introduction}

Laparoscopic pancreatic surgery has developed rapidly in recent years, and the reports of laparoscopic pancreatic resections, such as laparoscopic distal pancreatectomy and laparoscopic pancreaticoduodenectomy, have increased. (1-5) Although there are some problems including a complicated procedure, long operating time and high conversion rate, minimally invasiveness by laparoscopic approach is expected. (1-4) On the other hand, many benign and low grade malignant pancreatic lesions, including intraductal papillary mucinous neoplasms (IPMNs) and mucinous cystic neoplasms, have been detected by the improved diagnostic modalities, recently. Several surgical procedures for minimal resection of the pancreas, such as enucleation and middle pancreatectomy, to reserve the pancreatic parenchyma are considered for such benign and low grade malignant lesions. We report herein a case of successful laparoscopic middle pancreatectomy under a pancreatic duct-navigation surgery $(10,11)$ for a localized main pancreatic duct stenosis. 


\section{Surgical technique}

A 65-year-old Japanese woman was referred to our hospital from local clinic for the evaluation and treatment of an incidental detected localized stenosis of the main pancreatic duct (MPD). She presented with abdominal discomfort and a diagnosis of cholecystolithiasis was made at a local clinic. On admission, she had no complaint, and no abnormalities were found on physical examination. She had no history of alcohol consumption or abdominal blow. Preoperative blood examinations were within normal limits. Abdominal computed tomography (CT) and magnetic resonance pancreatography (MRP) demonstrated a localized stenosis of the MPD in the pancreatic body and a slight dilatation of the MPD in the tail side. No tumor lesion was detected by abdominal CT. Endoscopic retrograde pancreatography (ERP), similarly, showed the narrow segment and slight dilatation of the MPD in the pancreatic body and tail, respectively (Figure 1). A brushing cytology of the stenotic site showed Class 3. Because malignant possibility was not completely excluded, the patient was considered to be a candidate for surgery under a sufficient informed consent.

Under general anesthesia, a 5F-size endoscopic naso-pancreatic drainage (ENPD) tube (Olympus, Tokyo, Japan) was inserted into the main pancreatic duct to identify the narrow segment of the MPD by intraoperative pancreatography. Then the patient was placed in a spine position, and a $10 \mathrm{~mm}$ trocar was placed on the umbilicus, through which pneumoperitoneum was established. Other three $10 \mathrm{~mm}$ trocars were placed on midway between xiphoid and umbilicus, and right and left side of umbilicus on midclavicular line. Two 5mm trocars were placed on bilateral subcostal area.

After cholecystectomy, the gastrocolic ligament was divided and the pancreas was explored. The narrow segment of the MPD was showed by the intraoperative 
pancreatography through the ENPD tube (Figure 2A), and we marked the stenotic site with a clip. Upper and lower border of the pancreas was dissected and the dorsal surface of the pancreas was bluntly dissected from the splenic artery and vein, and a 6-mm Penrose drain was placed on the back side of the pancreas. The pancreas was encircled by a lead string on the proximal dividing line and by a Penrose drain on the distal dividing line, each dividing line were decided with $20 \mathrm{~mm}$ surgical margin from the narrow segment. Intraoperative pancreatography was performed again to confirm the position of the narrow segment and the dividing lines (Figure 2B), and then the pancreatic resection was performed. After the ENPD tube was removed, the proximal side of the pancreas was transected using an auto suture (Echelon 60-4.5; Ethicon Endo-Surgery Industries, Cincinnati, $\mathrm{OH}$ ), and the distal side of the pancreas was transected using a SonoSurg (Olympus, Tokyo, Japan) (Figure 2C). After pancreatic resection, a 6-cm mini-laparotomy was made in the upper midline of the abdomen and the resected specimen was delivered through the incision, and proximal and distal stumps of the pancreas were confirmed that there were no malignancies in intraoperative histopathologic diagnosis. The reconstruction procedures were performed through the small midline incision. The proximal jejunum was transected using an auto suture (Echelon 60-3.5) and the distal end of the jejunum was delivered through a window in the transverse mesocolon. An end-to-side pancreaticojejunostomy was performed with a 5F-size stenting tube and side-to-end jejunojejunostomy was performed on the $40 \mathrm{~cm}$ anal side from the pancreaticojejunostomy. Operating time was $630 \mathrm{~min}$ and blood loss was $100 \mathrm{~mL}$. A histological examination showed Ductal fibrosis and focal parenchymal fibrosis with focal pancreatic intraepithelial neoplasia IA. Postoperatively, severe complications, such as postoperative hemorrhage, pancreatic 
Kitasato

fistula, intraabdominal abscess, pulmonary complications, and deep vein thrombosis were not observed. The patient was discharged postoperative on $26^{\text {th }}$ day. 


\section{Discussion}

Recent developments of diagnostic modalities have increased detection of many benign and low malignant pancreatic lesions, and then various minimally invasive surgeries and/or pancreatic function preserving surgeries have been devised for such lesions. Reports of not only laparoscopic distal pancreatectomy but also laparoscopic pancreaticoduodenectomy have increased along with the recent development of laparoscopic pancreatic surgeries, and their low invasiveness has become to be admitted (1-4). Moreover, improvements of surgical devices and advances in knowledge of pancreatic anatomy have allowed surgeons to approach minimal resection of the pancreas purposing to reserve the pancreatic functions. Several pancreatic function preserving surgeries, including enucleation, pancreatic segmental resection of the head of the pancreas, or middle pancreatectomy, are being performed according to location, size and number of the benign and low malignant pancreatic lesions (6-9). In the present case, the patient had a localized stenosis of the MPD which not accompanying any detectable tumorous lesion, but the brushing cytology from the stenotic lesion was Class 3, suspicious. For such lesion, conventional pancreatic surgeries such as pancreaticoduodenectomy or distal pancreatectomy might become excessive invasiveness, and it is necessary to pursue low invasiveness and function preservation without loss of radicality. Then, we selected a laparoscopic surgery to minimized invasiveness, a middle pancreatectomy to reserve the pancreatic function along with completely resection of the lesion, and an ENPD stent-guided surgery $(10,11)$ to identify the stenotic lesion of the MPD following to a sufficient informed consent. In laparoscopic middle pancreatectomy, it is important to dissect bluntly the dorsal surface of the pancreas from the splenic vein and artery by using the advantage of laparoscopic 
Kitasato

magnified view. The insertion of an ENPD tube is useful for detecting the stenotic lesion of the MPD by intraoperative pancreatography which can be demonstrated repeatedly $(10,11)$. In our case, we completed the minimally invasive and function preserving surgery because intraoperative histopathologic diagnosis of the resected specimen indicated no malignancy, however, it is important to convert to the conventional resection in case of suggesting malignancy.

In conclusion, the laparoscopic middle pancreatectomy is effective to benign and low malignant pancreatic lesions in point of minimally invasiveness and function preservation. Additionally, the ENPD stent-guided surgery is useful to identify the lesion localized in the MPD by intraoperative pancreatography. 


\section{References}

1) Cunha AS, Rault A, Beau C, et al.: A single-institution prospective study of laparoscopic pancreatic resection. Arch Surg 2008; 143:289-295.

2) Mabrut JY, Fernandez-Cruz L, Azagra JS, et al.: Laparoscopic pancreatic resection: results of a multicenter European study of 127 patients. Surgery 2005; 137:597-605.

3) Melotti G, Butturini G, Piccoli M, et al.: Laparoscopic distal pancreatectomy: results on a consecutive series of 58 patients. Ann Surg 2007; 246:77-82.

4) Palanivelu C, Jani K, Senthilnathan P, et al.: Laparoscopic pancreaticoduodenectomy: technique and outcomes. J Am Coll Surg 2007; 205:222-230.

5) Dulucq JL, Wintringer P, Mahajna A: Laparoscopic pancreaticoduodenectomy for benign and malignant disease. Surg Endosc 2006; 20:1045-1050.

6) Hirono S, Yamaue $\mathbf{H}$ : Middle pancreatectomy for pancreatic neoplasms. J Hepatobiliary Pancreat Sci 2010; 17:803-807.

7) Takada T, Yasuda H, Amano $\mathbf{H}$, et al.: A duodenum-preserving and bile duct-preserving total pancreatic head resection with associated pancreatic duct-to-duct anastomosis. J Gastrointest Surg 2004; 8:220-224.

8) Nakagohri T, Kenmochi T, Kainuma $\mathbf{O}$, et al.: Inferior head resection of the pancreas for intraductal papillary mucinous tumors. Am J Surg 2000; 179:482-484.

9) Sata N, Koizumi M, Tsukahara M, et al.: Single-branch resection of the pancreas. J Hepatobiliary Pancreat Surg 2005; 12:71-75.

10) Kuroki T, Tajima Y, Tsutsumi R, et al.: Endoscopic naso-pancreatic stent-guided single-branch resection of the pancreas for multiple intraductal papillary mucinous 
Kitasato

adenomas. World J Gastroenterol 2006; 12:7203-7205.

11) Kuroki T, Tajima Y, Tsuneoka N, et al.: Combined pancreatic rsection and pancreatic duct-navigation surgery for multiple lesions of the pancreas: intraductal papillary mucinous neoplasm of the pancreas concomitant with ductal carcinoma of the pancreas. Hepatogastroenterology 2008; 55:1833-1836. 


\section{Figure legend}

Figure 1

A. MRP demonstrated a localized stenosis of the MPD in the pancreatic body (arrow head) and a slight dilatation of the MPD in the tail side (arrow).

B. Similarly, ERP showed the narrow segment (arrow head) and slight dilatation (arrow) of the MPD in the pancreatic body and tail, respectively.

\section{Figure 2}

A. Intraoperative pancreatography through the ENPD tube showed the narrow segment of the MPD.

B. A lead string (arrow) encircled the pancreas on the proximal dividing line and a Penrose drain (arrow head) encircled on the distal dividing line.

C. After middle pancreatectomy. The proximal side of the pancreas was transected by an auto suture (arrow), and the distal side of the pancreas transected using a SonoSurge (arrow head). SV: splenic vein. 
Figure 1
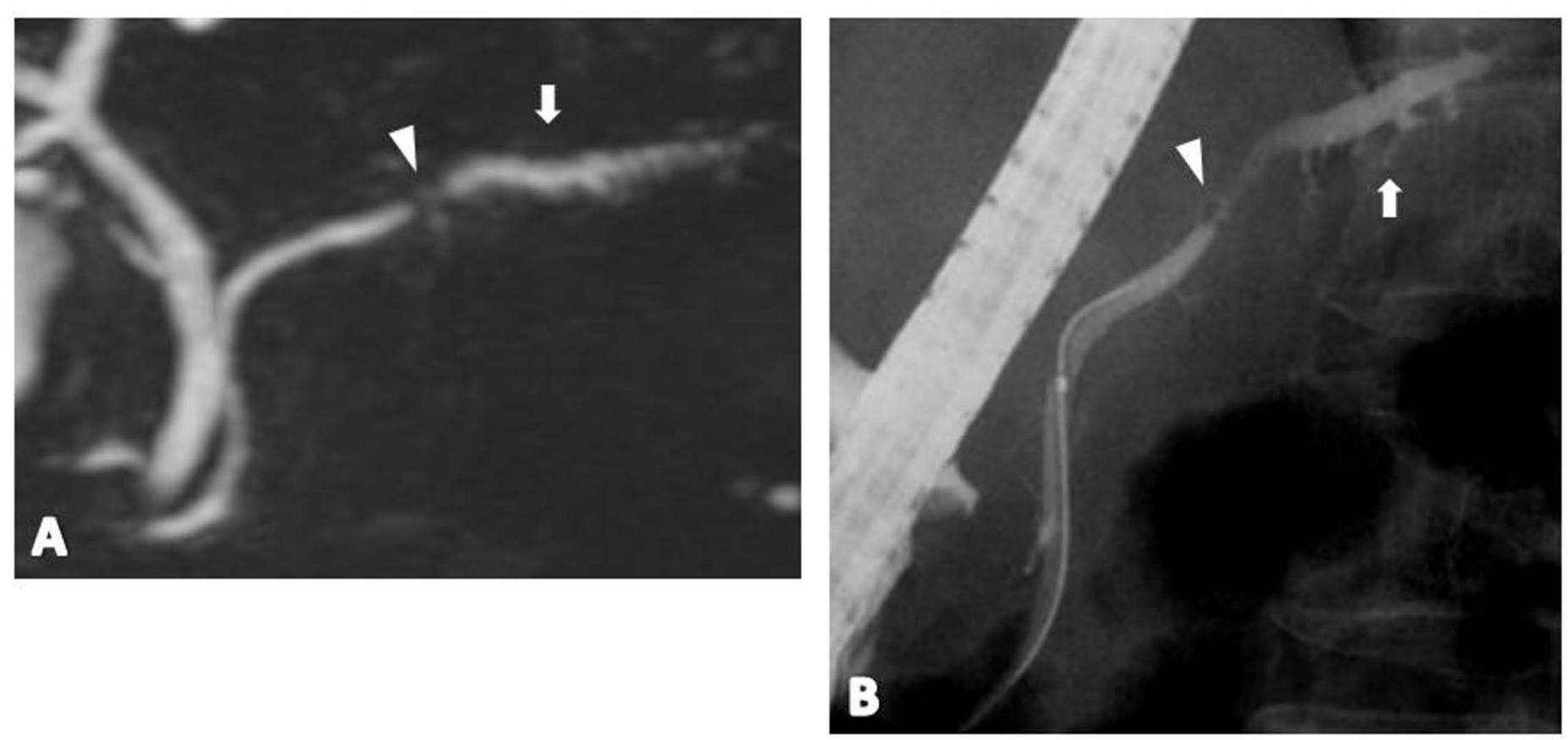
Figure 2
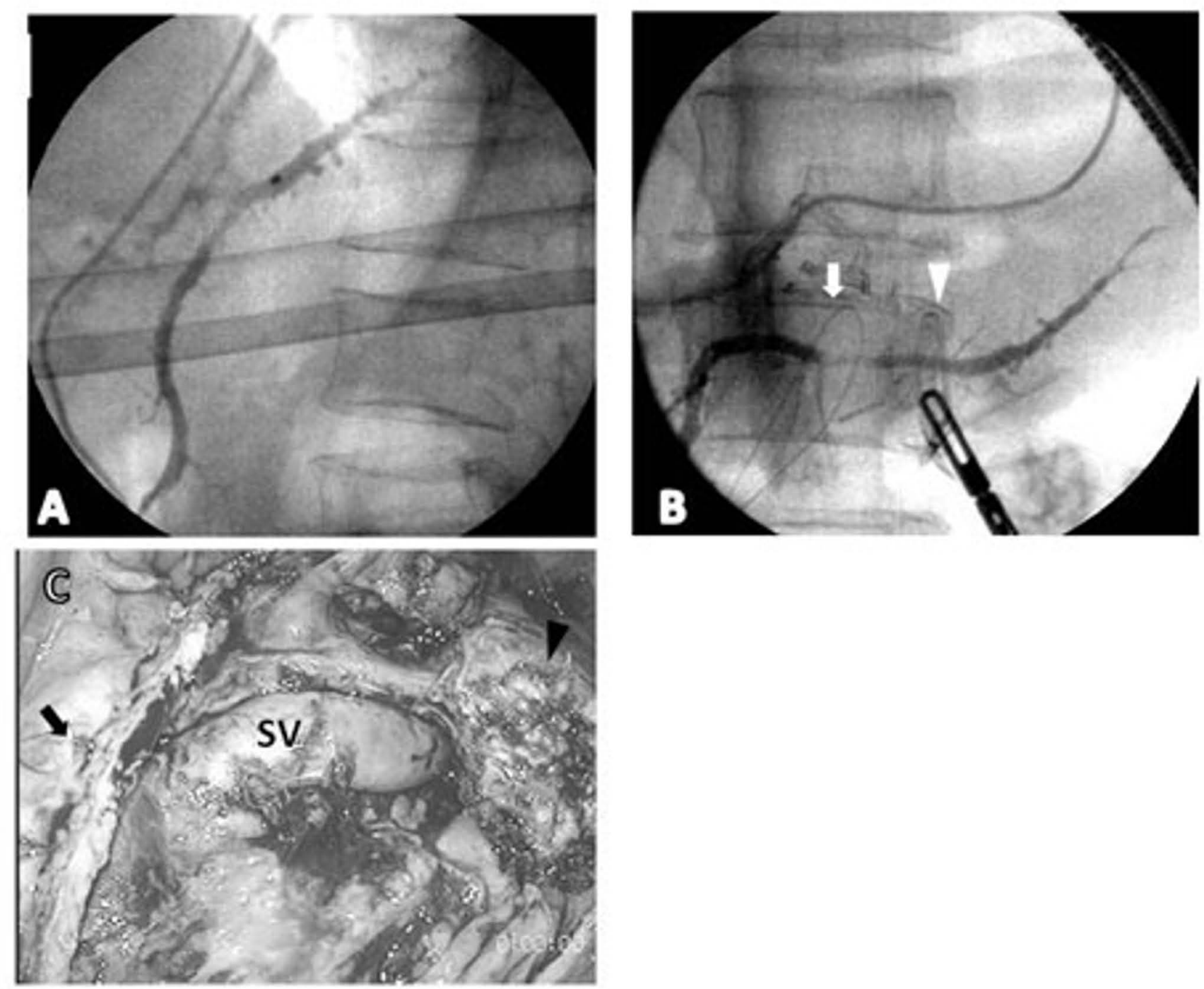